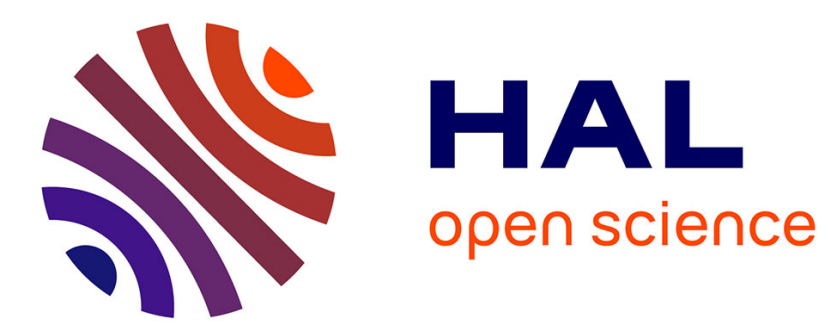

\title{
Décrypter l'activité délibérative dans la régulation territoriale par la négociation
}

Sophie Allain

\section{To cite this version:}

Sophie Allain. Décrypter l'activité délibérative dans la régulation territoriale par la négociation.

Négociations, 2009, 2, pp.229-243. 10.3917/neg.012.0229 · hal-01191158

\author{
HAL Id: hal-01191158 \\ https://hal.science/hal-01191158
}

Submitted on 29 May 2020

HAL is a multi-disciplinary open access archive for the deposit and dissemination of scientific research documents, whether they are published or not. The documents may come from teaching and research institutions in France or abroad, or from public or private research centers.
L'archive ouverte pluridisciplinaire HAL, est destinée au dépôt et à la diffusion de documents scientifiques de niveau recherche, publiés ou non, émanant des établissements d'enseignement et de recherche français ou étrangers, des laboratoires publics ou privés. 


\title{
DÉCRYPTER L'ACTIVITÉ DÉLIBÉRATIVE DANS LA RÉGULATION TERRITORIALE PAR LA NÉGOCIATION
}

\author{
Sophie Allain
}

De Boeck Université | Négociations

\author{
$2009 / 2-n^{\circ} 12$ \\ pages 229 à 243
}

ISSN 1780-9231

Article disponible en ligne à l'adresse:

http://www.cairn.info/revue-negociations-2009-2-page-229.htm

Pour citer cet article :

Allain Sophie, « Décrypter l'activité délibérative dans la régulation territoriale par la négociation », Négociations, 2009/2 n 12, p. 229-243. DOI : 10.3917/neg.012.0229

Distribution électronique Cairn.info pour De Boeck Université.

() De Boeck Université. Tous droits réservés pour tous pays.

La reproduction ou représentation de cet article, notamment par photocopie, n'est autorisée que dans les limites des conditions générales d'utilisation du site ou, le cas échéant, des conditions générales de la licence souscrite par votre établissement. Toute autre reproduction ou représentation, en tout ou partie, sous quelque forme et de quelque manière que ce soit, est interdite sauf accord préalable et écrit de l'éditeur, en dehors des cas prévus par la législation en vigueur en France. II est précisé que son stockage dans une base de données est également interdit. 


\title{
Décrypter l'activité délibérative dans la régulation territoriale par la négociation
}

\section{De Boeck Université | Négociations}

\author{
$2009 / 2-n^{\circ} 12$ \\ pages 229 à 243 \\ ISSN 1780-9231
}

\section{Article disponible en ligne à l'adresse:}

http://www.cairn.info/revue-negociations-2009-2-page-229.htm

\section{Pour citer cet article}

"Décrypter l'activité délibérative dans la régulation territoriale par la négociation", Négociations, 2009/2 n 12, p. 229-243.

Distribution électronique Cairn.info pour De Boeck Université.

(C) De Boeck Université. Tous droits réservés pour tous pays.

La reproduction ou représentation de cet article, notamment par photocopie, n'est autorisée que dans les limites des conditions générales d'utilisation du site ou, le cas échéant, des conditions générales de la licence souscrite par votre établissement. Toute autre reproduction ou représentation, en tout ou partie, sous quelque forme et de quelque manière que ce soit, est interdite sauf accord préalable et écrit de l'éditeur, en dehors des cas prévus par la législation en vigueur en France. II est précisé que son stockage dans une base de données est également interdit. 


\title{
Décrypter l'activité délibérative dans la régulation territoriale par la négociation
}

\author{
Sophie Allain * \\ INRA (UMR SAD-APT)
}

\begin{abstract}
En France comme au plan international, un principe participatif s'est progressivement imposé dans l'action publique territoriale. Qu'il s'agisse de planification urbaine ou environnementale, ou encore de projets d'aménagement ou d'infrastructure, il s'agit désormais d'impliquer les acteurs concernés dans les processus décisionnels en les invitant à débattre de lignes d'action engageant le collectif. On se propose ici de montrer l'intérêt d'une approche en terme de négociation pour décrypter l'activité délibérative à l'œuvre dans ces processus. Une telle perspective appelle des explications dans le contexte français où délibération et négociation tendent à être pensées de manière disjointe, voire en opposition. On mènera pour cela une discussion à la fois théorique et pratique visant à montrer que les lignes de clivage sont loin d'être aussi claires que cela. On précisera alors comment on utilise la négociation dans l'analyse d'activités délibératives en expliquant que la négociation a un intérêt avant tout comme concept d'investigation. Enfin, on appliquera cette approche à un cas concret en montrant ce que décrypter une activité délibérative par la négociation signifie et apporte. On s'appuiera tout au long de cet article sur un cas d'activité délibérative typique, a priori très éloigné de la négociation : le débat public organisé sous l'égide de la Commission National du Débat Public (CNDP) française, qui permet la confrontation d'arguments entre un maître d'ouvrage et un public à propos d'un projet d'infrastructure ; créée par la loi du 2 février 1995 relative au renforcement de la protection de l'environnement, cette procédure a été élargie par la loi du 27 février 2002 relative à la démocratie de proximité.
\end{abstract}

\section{DÉLIBÉRATION ET NÉGOCIATION : DU GLIVAGE DES CONGEPTS AU BROUILLAGE DES LIGNES}

\section{Un clivage prégnant entre délibération et négociation}

Appréhender une activité délibérative sous l'angle de la négociation appelle des explications, pour un objet qui tend à être enfermé par maints spécialistes dans un

* sophie.allain@agroparistech.fr 
modèle de la délibération fondé sur le paradigme dit de la « conversation » (Gary Remer, 2000 ; Philippe Urfalino, 2005) ou de la « discussion » (Sylvain Lavelle, 2007).

Cette conception de la délibération s'est développée sous l'influence de la pensée de Jürgen Habermas. Rappelons que pour ce philosophe (Habermas, 1997), dans les sociétés contemporaines complexes où il n'existe plus de principe universel pouvant fonder la légitimité de l'action publique, ce n'est qu'en permettant aux citoyens d'exercer pleinement leur autonomie civique et par la confrontation de leurs arguments qu'il est possible de favoriser la formation d'une opinion commune sur une ligne d'action engageant le collectif. Dès lors que la légitimité de l'action publique ne peut être que procédurale, c'est sur la création d'une structure de communication ouverte, permettant un échange libre d'arguments entre des participants égaux, que doit, selon lui, porter l'attention.

Au plan pratique, ce modèle imprègne de fait la philosophie d'action d'une instance comme la CNDP. Les principes idéaux de délibération énoncés par Habermas - ouverture et liberté des échanges, égalité des participants, «force du meilleur argument »-se retrouvent dans les règles d'organisation des débats publics édictées par la CNDP : publicité et transparence, équivalence de traitement entre les participants et impératif d'argumentation.

Or, une telle conception de la délibération amène à penser cette notion en contraste avec la négociation (Urfalino, 2005). Ainsi en est-il chez Habermas (1987) tout d'abord qui distingue l'« agir communicationnel » de l'« agir stratégique » : le premier concept s'applique à " l'interaction d'au moins deux sujets capables de parler et d'agir qui engagent une relation interpersonnelle [...]. (Ces acteurs) recherchent une entente sur une ligne d'action, afin de coordonner consensuellement leurs plans d'action et par là-même leurs actions » (p. 102). Le concept d'“ agir stratégique » est, lui, une extension du concept d'« agir téléologique » (p. 101) qui décrit un acteur choisissant les moyens qu'il juge efficace pour parvenir à un but ; dans le modèle stratégique, l'acteur fait intervenir dans son calcul les agissements d'au moins un acteur supplémentaire. Cette seconde conception de l'action est précisément celle qui est à la base de la théorie des jeux et du champ de la négociation tel que le définit Thomas Schelling (1986) : en effet, pour cet auteur, ce champ correspond aux situations de comportements stratégiques dans lesquelles chacune des parties « sait parfaitement que ses propres actes sont observés et peuvent entraîner des anticipations de la part de son adversaire, et se trouve donc conduit à agir en fonction de la situation potentielle qu'il se trouve amené à créer » (p. 37).

Mais cette distinction se retrouve chez de nombreux théoriciens contemporains de la délibération : ainsi, Jon Elster (1998) différencie l'« argumentation » qui est, selon lui, l'essence de la délibération et le « marchandage » par concessions mutuelles (bargaining ou log-rolling). Cela s'explique dans le contexte institutionnel américain où les processus de marchandage occupent une place importante et où la délibération apparaît comme une alternative plus morale pour fonder l'accord (Bernard Manin, 2002). Toutefois, une telle distinction tend aujourd'hui à être considérée comme allant de soi : Paul Magnette (2004), par exemple, explique 
qu'il se fonde sur "l'opposition conceptuelle entre délibération et négociation » pour analyser le processus d'élaboration d'un «projet de traité établissant une constitution pour l'Europe » par la « Convention européenne » en 2003.

\section{Un clivage en réalité très flou}

Un tel clivage conceptuel ne résiste cependant pas à l'examen empirique, et le brouillage des lignes qui en résulte invite à un dépassement théorique.

L'observation d'un débat public ${ }^{1}$ montre ainsi qu'une telle manifestation est très éloignée d'une situation idéale de délibération : en effet, le public actif est d'abord un public intéressé, venant pour défendre des intérêts et des points de vue sinon personnels du moins propres à un groupe particulier (les habitants de tel village ou de tel quartier ; les chefs d'entreprise ; les APNE...) et une large partie de l'auditoire ne s'exprime pas. Si les participants peuvent formuler des arguments, ils expriment aussi des opinions, des positions, des menaces. En fait, ils cherchent plus à convaincre les autres du bien-fondé de leurs points de vue qu'ils ne visent à s'entendre sur une ligne d'action commune. Souvent, les arguments semblent se croiser sans réellement se rencontrer, et le débat peine à progresser. Les échanges sont loin de relever du seul registre rationnel : un débat public, c'est aussi l'irruption de l'émotion, l'expression de peurs, de craintes, de colères, de rancœurs. Les participants s'accusent fréquemment les uns les autres de se mentir, de dissimuler ou travestir des informations, ces attaques étant en particulier dirigées contre le maître d'ouvrage.

Des observations de ce type ont conduit plusieurs auteurs (Remer, 2000 ; Urfalino, 2005 ; Lavelle ; 2007) à remettre en cause le modèle de la conversation qui imprègne l'approche de la délibération, en faisant valoir que ce modèle ne rendait pas bien compte des échanges discursifs à l'œuvre dans un processus délibératif. Remer (2000), en particulier, a proposé d'appréhender ceux-ci selon le modèle de la rhétorique ( «oratory »), revenant par là aux conceptions de la délibération en vigueur chez les penseurs de l'Antiquité : ce modèle assume en effet une asymétrie et une inégalité de fait entre les participants (peu d'orateurs face à un large auditoire ; compétences oratoires variables) ; en outre, se basant sur l'idée de persuasion plutôt que d'argumentation rationnelle, il reconnaît l'importance du pathos (le recours aux émotions) et de l'éthos (qui est l'orateur) à côté du logos, et présuppose la dimension agonistique des interventions - il s'agit de l'emporter contre les autres - et non la sincérité des orateurs (ceux-ci utilisent les arguments qu'ils estiment les plus efficaces, pas forcément ceux auxquels, à titre personnel, ils croient le plus).

Or, si l'on admet l'intérêt du modèle de la rhétorique pour rendre compte des échanges discursifs dans une délibération, la distinction conceptuelle entre délibération et négociation s'estompe : en effet, la négociation fait largement appel à

1. On s'appuie ici sur nos propres observations des débats publics de Charlas (projet de barrage) en 2003 et de la Francilienne (projet d'autoroute) en 2006. 
la «stratégie du verbe », pour reprendre cette expression heureuse de Gilbert Millet (1981), et use d'une diversité d'arguments relevant de plusieurs registres visant tout autant à justifier des positions et des points de vue qu'à influencer et contraindre l'autre.

En outre, dès lors que l'on n'assimile plus la délibération à un pur registre d'argumentation rationnelle, c'est non seulement la nature des échanges discursifs en jeu que l'on est amené à examiner sous un angle nouveau, mais aussi et plus fondamentalement les finalités de la délibération elle-même. En effet, dans la conception habermassienne comme chez les théoriciens de la « démocratie délibérative » qui s'intéressent à la délibération dans l'optique d'une amélioration du fonctionnement de la démocratie, seuls des échanges reposant sur une argumentation rationnelle permettraient une poursuite désintéressée du bien commun et donc de réaliser l'idéal démocratique ; il s'agit donc de mettre en place les conditions nécessaires à une telle forme de délibération pour ne pas laisser opérer le libre jeu social où prévalent des rapports de force inégaux et où chacun ne cherche qu'à satisfaire des intérêts particuliers. On suppose donc implicitement que ce n'est qu'en l'absence d'enjeux personnels que des individus sont en mesure de délibérer de manière morale. Mais, des individus peuvent-ils réellement être motivés à participer à une activité de délibération dans laquelle ils n'ont pas d'enjeu direct ? Certes, de telles situations peuvent se rencontrer dans le cadre professionnel c'est ainsi que fonctionnent les comités d'experts, par exemple. Mais, la discussion porte ici sur une participation élargie, en particulier sur celle des « simples » citoyens, dans le cadre de l'action publique en général. Dans cette perspective, il est utopique de considérer qu'une activité de délibération ne répond à l'idéal démocratique que si elle implique des individus n'ayant pas d'enjeu dans celle-ci, tout simplement parce qu'alors une grande partie de leurs raisons de participer disparaît ; certes, certains ont un intérêt pour la « chose publique » en soi, mais la majorité du public n'aspire pas à participer à la délibération publique, comme le notait déjà Walter Lippmann (1993) en 1925.

On peut ajouter que si la finalité d'une activité délibérative est de chercher à apprécier le bien commun pour juger du bien-fondé d'une ligne d'action publique, on peut douter qu'une argumentation purement rationnelle parvienne à atteindre un tel objectif. Si la délibération publique doit en effet permettre de fonder la légitimité de l'action publique dans un contexte où il n'existe plus de principe universel susceptible de s'imposer à tous et où coexistent et s'affrontent des légitimités d'ordres variés, ce n'est pas en faisant comme s'il était possible de faire abstraction de ce pluralisme que l'on peut espérer résoudre le problème. C'est au contraire en prenant acte de la diversité de points de vue qu'implique le pluralisme et en exploitant cette richesse cognitive que l'on peut faire émerger la multiplicité des enjeux associés à une action et de là tenter de les intégrer dans un projet qui peut alors prétendre répondre au bien commun. Dans cette optique, un argument n'est pertinent que s'il est intéressé c'est-à-dire référé à une situation concrète en rapport avec l'action envisagée. Cette discussion suggère qu'il n'y a pas de raison au plan théorique de distinguer « action stratégique » et «action communicationnelle » dans une situation de délibération publique : les échanges verbaux à l'œuvre dans ce type de situation sont un moyen (parmi d'autres) qu'utilisent des acteurs en si- 
tuation d'interdépendance lorsqu'ils prennent conscience qu'ils ne peuvent atteindre leurs objectifs sans tenir compte des autres, ou encore qu'ils ont quelque chose à gagner en le faisant ou à perdre en ne le faisant pas. II convient seulement de ne pas faire de contresens sur la notion d' "action stratégique » : comme l'a souligné Erhard Friedberg (1993), si celle-ci présuppose un comportement calculateur, le terme de calculateur ne doit pas être entendu au sens restreint de froid et égoïste, mais dans un sens beaucoup plus large : il signifie que tout acteur a un comportement motivé par une visée et qu'il a de « bonnes raisons » d'agir comme il le fait.

On peut d'ailleurs constater que le débat public n'est pas une procédure conçue pour développer la participation du public et améliorer la vie démocratique, mais pour répondre à l'insatisfaction à l'égard de l'enquête publique et aux situations d'impasse constatées par les grands maîtres d'ouvrage publics (Blatrix, 2007). Cette procédure prend ainsi acte du caractère nécessaire de la délibération publique dans un contexte où un porteur de projet n'est pas en mesure de mener à bien seul son action.

S'il n'apparaît plus aussi pertinent d'appréhender l'activité de délibération seIon le cadre théorique de l'argumentation rationnelle proposé par les théoriciens de la « démocratie délibérative », est-il pour autant approprié de mobiliser le cadre théorique de la négociation et jusqu'où ? Revenons tout d'abord pour cela sur les limites habituellement prêtées à la négociation. Celle-ci correspondrait à un régime d'interaction dans lequel chacun ne recherche que la satisfaction des intérêts qu'il défend, et ne pourrait aboutir qu'à des compromis résultant de concessions mutuelles selon une logique gagnant - perdant. Or, dès lors que l'on se situe dans des situations de négociation complexes engageant un grand nombre de parties prenantes, la structuration du jeu que suppose ce type d'échanges (positions bien définies ; absence de volonté de coopération au-delà de la transaction concernée) n'existe pas : comme l'a fait observer William Zartman (1994), dans les négociations multilatérales, la multiplicité des questions induit une dynamique d'interactions beaucoup plus ouverte parce qu'elle empêche une polarisation stricte du jeu, même si des coalitions se créent, et parce qu'elle est source d'innovation. On peut ajouter que si Urfalino (2005, p. 110) considère que le propre d'une délibération est que " tous les participants n'aient pas déjà leur volonté complètement déterminée et fixée et que, partant, les discours échangés soient susceptibles de contribuer à la formation de la détermination de certaines de ces volontés ", une telle propriété s'applique largement aux négociations multilatérales dans laquelle les positions de chacun sont loin d'être arrêtées. Enfin, dans des contextes où les protagonistes sont amenés à maintenir des relations dans la durée - cas de l'action publique territoriale -, les protagonistes ne peuvent interagir comme s'ils étaient uniquement des adversaires et sont enclins à coopérer au moins un minimum ${ }^{2}$. Autrement dit, si l'on ne restreint pas la négociation à un régime de marchandage,

2. Dean Pruitt et Peter Carnevale (1993) ont largement souligné que, dans la plupart des négociations, les parties tiennent aussi compte des intérêts des autres parties (other interest) et pas seulement de leurs intérêts propres. 
qui correspond à la vision stéréotypée de la négociation que proposent maints théoriciens, les critiques à son encontre ne tiennent pas.

Au total, dès lors qu'on dissocie délibération et argumentation rationnelle, qu'on constate qu'il n'est pas si aisé de distinguer argumentation et persuasion et qu'on reconnaît l'existence de plusieurs régimes de négociation, les frontières entre délibération et négociation se brouillent au plan théorique. II convient maintenant d'aller plus loin et de montrer l'intérêt du cadre théorique de la négociation pour analyser les situations de délibération publique dans l'action publique territoriale.

\section{LA NÉGOCIATION COMME CONCEPT D'INVESTIGATION D'UN DÉBAT PUBLIC SUR UN PROJET D'INFRASTRUCTURE}

Les discussions précédentes relatives à la délibération ont été engagées par des auteurs cherchant à analyser ce qui se joue dans une activité délibérative ${ }^{3}$. L'objectif poursuivi ici n'est cependant pas tant de savoir comment rendre compte de ce type d'activité de la façon la plus juste possible que de savoir comment mettre en évidence ses apports. En effet, par la tribune qu'elle offre et par le brassage d'arguments qu'elle permet, une activité délibérative comme le débat public ouvre un espace d'incertitudes ; les maîtres d'ouvrage ne s'y trompent d'ailleurs pas, qui redoutent souvent fortement ce moment d'épreuve publique ${ }^{4}$. Le débat public comporte ainsi un potentiel transformatif, et on avance ici qu'une lecture en terme de négociation est la mieux à même d'appréhender ce potentiel. On montrera auparavant comment on peut représenter un débat public comme une négociation.

Le débat public : une séquence de négociation à orientation distributive " médiée "

La structure d'un débat public sur un projet d'infrastructure montre que celle-ci, du fait même de la procédure, présente en fait bien des traits d'une « négociation de projet » (Dupont, 1994, p. 357) dans laquelle un maître d'ouvrage cherche à rendre acceptable son projet auprès des acteurs concernés : en effet, le projet est présenté au public par un maître d'ouvrage qui, à travers le processus de concep-

3. Dans cette optique, Lavelle (2007, p.364), par exemple, a proposé de recourir à « une typologie combinatoire [...] pour caractériser la diversité des compositions politiques propre à la dynamique d'un débat public ".

4. Dans son étude sur le Bureau d'Audiences Publiques sur l'Environnement (BAPE), structure québecoise dont s'inspire la CNDP, Louis Simard (2006) a montré, en s'appuyant sur le cas de l'entreprise Hydro-Québec, qu'un maître d'ouvrage s'efforce d'organiser des concertations avec les acteurs concernés et de se mettre d'accord avec ceux-ci pour éviter d'avoir à se soumettre à une audience publique, procédure correspondant à notre débat public. 
tion de l'ouvrage, a déjà effectué des choix, écarté d'autres options, et se retrouve naturellement plus dans l'attitude de défendre une position (ou un éventail de positions) que d'échanger avec d'autres en vue de mettre au point une ligne d'action commune. Certes, il s'agit d'une négociation incomplète puisque l'issue de celleci est extérieure au débat public - le maître d'ouvrage prend sa décision ultérieurement -, mais le débat public peut être considéré comme une séquence de négociation, à orientation distributive : il peut en effet être décrit comme un jeu d'influences mutuelles dans lequel chacun cherche à faire progresser la situation à son avantage vis-à-vis de l'enjeu que constitue la décision relative au projet, dans une situation d'interdépendances où nul ne peut imposer son point de vue, y compris le maître d'ouvrage (les acteurs locaux peuvent disposer d'une force de blocage en se mobilisant suffisamment et les collectivités territoriales sont souvent parties prenantes du financement du projet). L'orientation est distributive dans la mesure où chacun semble chercher à l'emporter contre l'autre, mais la plupart des protagonistes ne sont en réalité pas dans une logique pure de jeu gagnant -perdant, en particulier le maître d'ouvrage qui a tout intérêt à se prévaloir de l'acceptation sociale de son projet. Cette séquence de négociation présente des traits spécifiques au sein de la négociation englobante dans laquelle elle prend place: il s'agit d'un moment d'échanges directs et publics entre les protagonistes, à la différence des échanges qui peuvent avoir lieu antérieurement ou postérieurement et qui se tiennent dans des instances closes, de manière souvent confidentielle, et de façon bilatérale ou du moins entre un nombre réduit de participants. Le dispositif du débat public joue par ailleurs comme « instance de médiation » (Allain, 2005a) dans la mesure où il réduit les asymétries de pouvoir en favorisant l'accès à l'information et l'expression de tous ceux qui le souhaitent.

\section{Le débat public comme outil de structuration} d'une négociation de régulation

Chercher à appréhender le potentiel d'un débat public se situe pour nous dans la perspective d'une démarche d'investigation pragmatique, au sens de John Dewey (2003a). Dans cette perspective, on assume que le bien commun ne peut être qu'un idéal et qu'il se présente comme un chemin en construction dans un monde « en train de se faire » (in the making) ; il ne peut donc être que recherché et approché par expérimentation collective. Dans ses conditions, la tâche des sciences sociales n'est pas de déterminer ce qui pourrait être ou devrait être, mais d' « aider à la création de méthodes telles que l'expérimentation puisse se poursuivre de manière moins aveugle [...] (et) plus intelligente » (Dewey, 2003b, p. 75). II s'agit donc ici tout autant d'apprendre de l'expérience collective que constitue le débat public que de faire en sorte que celle-ci soit la plus fructueuse possible. On se place plus précisément du point de vue de la Commission Particulière du Débat $\mathrm{Pu}$ blic (CPDP) en charge du débat, puisque celle-ci doit à la fois organiser ce débat et en rendre compte dans un rapport public remis au maître d'ouvrage, rapport sur la base duquel celui-ci doit prendre sa décision quant aux suites à donner au projet.

L'activité d'une telle instance a tout intérêt à être vue comme une activité de médiation (Allain, 2007). En effet, une CPDP a un rôle actif qui va au-delà d'une 
activité de facilitation : il ne s'agit pas seulement de favoriser la prise de parole de ceux qui souhaitent intervenir et d'effectuer une « synthèse » du débat, mais aussi d'aider à rendre le débat productif et d'en fournir une représentation utile pour la poursuite du processus. Considérer la conduite d'un débat public comme une activité de médiation, et donc envisager le débat public comme une négociation, permet de prendre acte de ce rôle actif et de se donner les moyens de le jouer au mieux : se placer dans cette perspective, c'est reconnaître que chaque participant - qu'il s'agisse du maître d'ouvrage, d'un acteur institutionnel ou d'un citoyen « ordinaire »-, ne peut avoir qu'une vision partielle des enjeux du projet et de ses effets en fonction des intérêts qu'il défend et que seule la confrontation de ces différentes visions permet de faire émerger la négociation réelle à l'œuvre, la « négociation de régulation » (Allain, 2005b ; 2008a). En effet, si un débat public sur un projet d'infrastructure se présente de prime abord comme une négociation de projet, c'est une négociation à propos de la régulation de problèmes relatifs aux rapports qu'entretient l'homme avec son environnement dans un territoire qui est fondamentalement à l'œuvre, puisqu'un débat public ne porte pas que sur les caractéristiques d'un projet mais aussi sur son opportunité. Par exemple, si le projet soumis au débat porte sur la construction d'une portion d'autoroute, c'est le problème des transports dans un territoire qui est l'objet réel du débat, le projet représentant une solution parmi d'autres pour résoudre ce problème. Le terme de régulation ne renvoie pas tant ici à l'idée de production de règles qu'à celle d'un processus visant à corriger des dysfonctionnements et à assurer la pérennité d'un collectif ${ }^{5}$. Cette négociation de régulation est finalisée par la mise en place d'un dispositif d'ensemble qui se veut cohérent et organisateur pour les actions futures, et qui peut conduire à accepter le projet d'infrastructure initial, à l'adapter ou à le rejeter au profit d'une autre solution. Parce qu'elle met implicitement ou explicitement en jeu la fabrication d'une conception du bien commun territorial, elle peut engager des conflits de valeurs mais peut aussi contribuer à faire évoluer le collectif concerné vers une communauté au sens de Dewey. Elle suppose une construction collective du problème et des solutions, et est donc par essence à orientation intégrative ${ }^{6}$. Mais, elle aura d'autant plus de chances de favoriser l'émergence de solutions créatives et l'adoption de solutions gagnant-gagnant qu'elle se déroulera sur un mode collaboratif. Dans une telle conception, on considère alors que le rôle d'une CPDP est de favoriser la mise en évidence et la progression de cette «négociation de régulation " et son orientation vers un mode d'interaction collaboratif.

Une telle perspective suppose de disposer d'outils méthodologiques susceptibles d'aider à mener cette investigation. On a suggéré dans un précédent article (Allain, 2004) qu'il convenait pour cela d'équiper le concept de négociation, et on

5. On renvoie ici à la définition de la régulation proposée par B. Lecuyer (1986) - « processus d'ensemble qui contribue [...] à assurer le maintien et la permanence de la structure sociale »- et reprise par les politologues travaillant sur la régulation politique (Commaille et Jobert, 1998).

6. Rappelons que pour Walton et McKersie (1993), une négociation intégrative est une situation dans laquelle les parties s'efforcent de résoudre collectivement un problème d'intégration de leurs objectifs ; elle correspond à un processus de résolution conjointe de problème (joint problem-solving process) qui peut être un jeu à somme positive, au sens de la théorie des jeux. 
a notamment proposé un cadre analytique permettant d'appréhender le processus de négociation. On a récemment complété cette approche (Allain, 2008a) en définissant différents « régimes » possibles de négociation dans le domaine de l'action publique territoriale ${ }^{7}$, c'est-à-dire de modalités particulières de négociation caractérisables par différents critères. Ceux-ci se divisent en deux grandes catégories, selon qu'il est possible ou non de déceler le début d'un dialogue collectif pour traiter un problème faisant l'objet de points de vue divergents. Dans la première catégorie, les régimes se différencient principalement selon la finalité de la négociation et son orientation principale (distributive ou intégrative) ; on distingue en particulier le régime de la « négociation de régulation » du régime du « marchandage » qui correspond à une négociation à orientation distributive centrée sur la volonté de parvenir à un accord - sans souci de clarifier le problème sous-jacent et les éventuels différends - et reposant sur un processus de concessions mutuelles et de compensations. Dans la seconde catégorie, on définit par exemple un régime de "guerre froide ${ }^{8}$ dans lequel les protagonistes se voient comme des adversaires, voire comme des ennemis, et n'ont comme objectif que de gagner ou d'obtenir le plus possible - même au détriment des autres, ou encore de dissuader l'autre de poursuivre son action; les échanges s'effectuent à distance, de manière indirecte, ou sous forme de joutes oratoires. Se situant toujours à la lisière du conflit, et pouvant même basculer ponctuellement dans des épisodes conflictuels, ce régime se distingue cependant d'un pur conflit - dans lequel on sort de la négociation -, dans la mesure où les protagonistes veillent aussi à se manifester des signes de non agression et à maintenir de toute façon le principe d'un échange.

Muni de cet équipement, il est alors possible pour une CPDP de décrypter les échanges à l'œuvre dans un débat public pour repérer la négociation de régulation en jeu, l'aider à progresser au cours du débat, la mettre en évidence dans le compte-rendu du débat et favoriser l'établissement d'une négociation intégrative qui doit se poursuivre au-delà du débat. On va voir qu'il s'agit pour cela de construire un cadre cognitif rendant compte de cette négociation de régulation et de son état

7. Le champ de l'action publique territoriale souffre en effet d'un manque de références adaptées à ses spécifités, la littérature sur la négociation s'intéressant principalement aux champs consacrés des négociations commerciales, des négociations collectives et des négociations internationales. Or, si une négociation de régulation dans le domaine de l'action publique territoriale présente des similitudes avec la négociation collective dans le domaine du travail, par exemple, elle possède cependant plusieurs spécificités : le cadre des échanges n'est pas forcément bien défini au départ et peut se fabriquer avec la négociation, ne serait-ce que parce que la construction même de l'objet de la négociation suppose de circonscrire celui-ci territorialement; de même, elle ne dispose souvent pas de règles et de pratiques aussi bien établies, les participants n'ayant d'ailleurs pas réellement le sentiment de négocier ; enfin, fondamentalement multilatérale, elle implique des acteurs plus variés (représentants de l'Etat, élus, associations, usagers...) et plus hétérogènes, ceux-ci n'étant pas forcément institutionnalisés. Là où la négociation collective se présente souvent comme un affrontement de positions clairement définies entre patronat et syndicats, avec des jeux de rôles bien rôdés, la négociation de régulation dans le cadre de l'action publique territoriale offre une image beaucoup plus kaléidoscopique d'acteurs qui se découvrent mutuellement et s'affirment au gré du processus, de différends profondément ancrés comme de projets collectifs.

8. Cette dénomination s'inspire de l'image de « guerre limitée » de Schelling (1986, p. 19). 
d'avancement en s'appuyant sur les arguments échangés, et de repérer les conditions organisationnelles susceptibles de permettre la mise en place d'un dispositif de gouvernance garantissant la poursuite de la concertation au-delà du débat pour parvenir in fine à un accord de régulation. On s'appuiera pour cela sur le cas du débat public relatif au projet de réservoir de Charlas (Haute-Garonne), qui a eu lieu de septembre à décembre 2003.

\section{APPLICATION : DÉGRYPTAGE DU DÉBAT PUBLIC DE GHARLAS PAR LA NÉGOGIATION}

Le projet soumis au débat portait sur la construction d'une grande retenue (110 millions de $\mathrm{m}^{3}$ ) en dérivation de la Garonne, à l'amont de ce bassin (dans la région du Comminges), pour assurer une fonction de soutien d'étiage : deux tiers de l'eau fournie devaient réalimenter ce fleuve sur l'ensemble de son linéaire, et le tiers restant plus de $1000 \mathrm{~km}$ de ses affluents situés en rive gauche, notamment en Gascogne. Le projet était présenté par le Syndicat Mixte d'Etudes et d'Aménagement de la Garonne (SMEAG), établissement public territorial de bassin dont le territoire de compétences s'étend sur tout le linéaire du fleuve et qui regroupe les deux régions Aquitaine et Midi-Pyrénées et les quatre départements de Gironde, Haute-Garonne, Lot-et-Garonne et Tarn-et-Garonne. Un débat public avait était demandé dès 1997 par l'association de protection de la nature France Nature Environnement à la suite d'une résolution favorable du Comité de Bassin Adour-Garonne en 1996 pour la poursuite de l'étude du projet, malgré un avis défavorable de son conseil scientifique. Le débat public n'a cependant pu se tenir que fin 2003 en raison notamment d'incertitudes sur l'acteur public susceptible de présenter le projet et d'un dossier jugé incomplet par la CNDP. L'analyse présentée ici repose sur une étude approfondie des arguments produits au cours du débat (Allain, 2005b ; 2008b).

\section{Un régime apparent de guerre froide improductif}

Pour maints observateurs, le débat public de «Charlas » a été vécu comme une guerre de positions entre deux protagonistes principaux, le porteur de projet d'un côté et le Comité Contre Charlas, regroupant des riverains de plusieurs localités voisines du site, opposé au projet de l'autre. Les autres participants se sont ralliés à l'une ou l'autre de ces positions ou sont restés en retrait : ainsi, le SMEAG a été soutenu par la profession agricole, une petite association locale, Charlas 2000, désireuse de promouvoir le développement du site, et le Conseil Economique et Social de Midi-Pyrénées, tandis que le Comité Contre Charlas a été appuyé par un large collectif d'associations de protection de la nature et de consommateurs; l'État, l'Agence de l'Eau et la plupart des élus ne se sont pas prononcés sur le projet. Cette structuration est apparue dès la séance d'ouverture et n'a pas évolué ultérieurement, donnant l'impression que le débat n'avait servi qu'à organiser ou à renforcer les deux coalitions. Au total, la personne publique responsable du projet, le SMEAG, a considéré que le débat ne lui avait pas réellement permis de progres- 
ser dans son analyse, et reste à ce jour toujours dans l'indécision vis-à-vis du projet. Pourtant, on va voir que ce débat permettait de jeter les bases d'une négociation de régulation explicite. Une première étape est de reconnaître le conflit de régulation en jeu dans celui-ci.

\section{Un conflit de régulation}

Si le SMEAG s'est efforcé de minimiser l'opposition en faisant comme si celle-ci se limitait à celle de quelques riverains, reprenant ainsi le thème classique du syndrome NIMBY (Not In My Backyard) cher aux aménageurs, c'est en réalité une divergence de fond à propos de la régulation territoriale de l'eau qui est apparue : en effet, les partisans du projet n'ont cessé d'affirmé la nécessité de construire la retenue pour remédier aux déficits récurrents des rivières et respecter les normes de débit fixées par le Schéma Directeur d'Aménagement et de Gestion des Eaux (SDAGE), instrument légal de planification de l'eau à l'échelle du territoire d'une Agence de l'Eau issu de la loi sur l'eau de 1992 et adopté en Adour-Garonne en 1996 ; a contrario, les opposants ont en permanence fait valoir que les déficits existants témoignaient d'une situation de dérégulation chronique et appelé à repenser la gestion territoriale de l'eau, avançant qu'un projet alternatif reposant sur une mobilisation accrue des barrages-réservoirs hydro-électriques d'amont était possible. Les jeux de mots martelés sous forme de slogans par les protagonistes ont montré que cette divergence s'ancrait dans des conceptions radicalement différentes de valeurs a priori consensuelles comme le « développement durable » ou l'« intérêt général », révélant la profondeur du clivage : si les partisans du barrage ont ainsi vanté les qualités d'un projet d' “aménagement durable ", soulignant le caractère structurant et flexible de l'ouvrage envisagé, les adversaires ont critiqué un projet de " développement murable » reposant sur « une vision « tuyau » des rivières »; de même, là où les premiers ont vu un projet " pour le grand SudOuest ", susceptible de favoriser le développement économique de cette vaste région, les seconds ont dénoncé un projet qui « détruit un territoire », - le site envisagé pour la construction de la retenue -, en réduisant fortement son attrait pour les riverains (marnage, précipitations accrues...) et en privant les jeunes agriculteurs locaux de terres pour s'installer. Voyons comment les controverses qui se sont exprimées tout au long du débat pouvaient permettre de transformer ce conflit de régulation apparemment inextricable en une négociation de régulation explicite.

\section{Un nouveau cadrage cognitif du problème de régulation}

A travers la guerre de positions que les protagonistes se sont livrés, les partisans du barrage se sont efforcés de convaincre de la nécessité et de l'efficacité du projet pour améliorer la régulation territoriale de l'eau, les opposants cherchant de leur côté à contester celles-ci, à montrer le caractère inacceptable du projet, et à faire valoir la possibilité d'une solution alternative. Les arguments qu'ils ont échangés ont permis de mettre en évidence le cadre cognitif sur lequel reposait le projet et de faire émerger un cadre cognitif élargi pour repenser le problème de régulation en jeu. 
Le débat a en effet permis de comprendre que le cadre cognitif qui a fait concevoir le projet de réservoir de Charlas comme une nécessité reposait implicitement sur quatre présupposés :

- un état de déficit avéré de la Garonne au regard des normes de débit fixées par le SDAGE ;

- une demande en eau constante ou croissante sur le territoire envisagé (Garonne-Gascogne), du fait en particulier des besoins agricoles pour l'irrigation très développée dans cette région ;

- une offre en eau limitée, à cause notamment d'une possibilité réduite de mobilisation des réserves hydro-électriques d'amont (coût élevé et sécurité de fourniture incertaine dans un contexte de libéralisation du marché de l'énergie) ;

- un principe de régulation territoriale de l'eau reposant sur le couplage des territoires de Garonne et de Gascogne.

Les controverses ont permis de « détendre » ce cadre considéré comme fixe en remettant en cause chacun de ces présupposés, et d'ouvrir (ou rouvrir) par là l'espace des possibles en terme de dispositif de régulation:

- elles ont ainsi fait ressortir que les normes de débit fixées par le SDAGE résultent d'un compromis établi à un moment donné et qu'elles peuvent donc être révisées ;

- elles ont par ailleurs amené à questionner la place de l'irrigation dans le développement agricole régional : le maïs irrigué, culture très consommatrice en eau qui s'est fortement développée dans les années 1970 et qui est à l'origine de l'importante demande en eau agricole de la région, restera-t-il une culture rentable dans un contexte d'évolution de la politique agricole communautaire vers une réduction des soutiens de prix et une prise en compte accrue de la protection de l'environnement ? L'irrigation est-elle une condition de rentabilité pour l'agriculture du sud-ouest ? Peut-elle réellement contribuer au maintien de petites exploitations face à une tendance lourde de réduction du nombre des structures agricoles et d'accroissement de la taille de celles-ci ? Est-il raisonnable de fonder un modèle de développement agricole sur l'irrigation dans une région naturellement pauvre en eau comme la Gascogne?

- les controverses ont en outre conduit à suggérer qu'une mobilisation accrue des réserves hydro-électrique d'amont pouvait être concevable dans un contexte de renouvellement de nombreuses concessions donnant la possibilité de réviser les priorités de production et les modes d'exploitation de ces ouvrages ;

- elles ont également mis en évidence que si le projet était présenté comme un prolongement du " principe de solidarité » entre la Garonne et la Gascogne introduit avec la construction du Canal de la Neste, cette solidarité, dans une configuration ne mettant en œuvre des transferts d'eau que dans un sens, était loin d'être acceptée par les habitants du Comminges, et que le principe même de régulation reposant sur un couplage entre ces deux territoires n'allait pas de soi ; ne serait-il pas plus pertinent que chaque territoire assure son propre équilibre hydraulique? 
- enfin, la question du réchauffement climatique a introduit ou renforcé des interrogations sur l'avenir de l'agriculture et de l'irrigation, la pertinence des techniques actuelles de gestion d'eau (du fait de la fonte des glaciers, les barrages pourront-ils toujours stocker de l'eau ?) et l'évolution des consommations en énergie (accroissement possible de la demande estivale pour la climatisation).

Au total, le jeu des controverses a donc mis en exergue les présupposés sur lesquels se fondait la conception du projet, en même temps qu'il a favorisé la construction d'un cadre cognitif collectif élargi faisant émerger le système d'interdépendances à prendre en compte pour appréhender le problème de régulation en jeu. Sur le plan temporel, ce nouveau cadre invite à se projeter dans l'avenir en envisageant la possibilité de scénarios de rupture plutôt que la prolongation de tendances comme le faisait le cadre cognitif initial. Sur le plan territorial, il met en évidence la nécessité de raisonner à une échelle spatiale suffisamment large permettant d'appréhender l'ensemble des paramètres jouant sur l'offre et la demande en eau. Fait de questions plutôt que d'affirmations, il fait apparaître des zones d'incertitude, mais aussi des marges de manœuvre à explorer pour construire un nouveau dispositif de régulation, en invitant en particulier à mettre en rapport la gestion des usages et les règles de répartition de l'eau.

\section{Une carence de gouvernance}

En élargissant le cadre de réflexion et en générant de nouvelles questions, le débat a en même temps révélé des problématiques souffrant d'un «vide du politique » : c'est le cas du principe de solidarité sur lequel se fonde la régulation territoriale de l'eau, de la place de l'irrigation dans le sud-ouest, notamment en Gascogne, et de l'usage des barrages hydro-électriques. S'il a fait apparaître la nécessité de prospecter plus en avant ces trois questions et d'en débattre collectivement, il a également pointé une carence de gouvernance pour mener à bien cela : il a ainsi montré les limites des instruments de planification de l'eau pour traiter ces questions et mis en évidence les difficultés d'un acteur comme le SMEAG à assumer la responsabilité d'un tel projet. Par là même, il a pointé la nécessité d'inventer un nouveau cadre de concertation pour traiter ce problème de régulation et des pistes pour concevoir ce cadre.

\section{CONCLUSION}

Un certain nombre de théoriciens distinguent délibération et négociation et jugent inopportun d'appliquer le concept de négociation à l'analyse d'activités délibératives. Une discussion tant théorique qu'empirique des échanges discursifs en jeu et des finalités d'une activité délibérative visant à éclairer la décision publique telle que celle prévue par la procédure française de débat public, comme une compréhension de la notion de négociation ne se réduisant pas à l'idée de marchandage, montre les limites d'une telle orientation. Au contraire, on a montré le potentiel du 
concept de négociation pour appréhender le potentiel transformatif d'un débat public dès lors que l'on équipe celui-ci de façon à décrypter la négociation à l'œuvre. Une telle approche s'inscrit dans une démarche d'investigation pragmatique au sens de John Dewey dans laquelle on cherche à ce que l'expérience collective en jeu soit la plus fructueuse possible. Dans un débat public considéré comme une séquence de négociation à orientation distributive "médiée », il s'agit ainsi de mettre en évidence la négociation de régulation sous-jacente et son état d'avancement. Appliquée à un débat public comme celui de Charlas où l'opportunité du projet était fortement contestée, l'analyse des arguments échangés a permis de construire un cadre cognitif élargi faisant apparaître le système d'interdépendances à prendre en compte pour appréhender de manière nouvelle le problème de régulation en jeu et jeter les bases d'une négociation de régulation explicite.

Si on a choisi ici le cas emblématique du débat public pour montrer l'intérêt du concept de négociation dans l'analyse d'activités délibératives, l'approche peut a fortiori être étendue à des concertations territoriales diverses, que celles-ci entrent dans le cadre de procédures formalisées ou dans le cadre de dispositifs informels et s'appliquent à des projets d'aménagement ou à des démarches de planification. Elle peut ainsi permettre de dépasser les limites souvent prêtées à la concertation (enlisement du dialogue, lien ambigu ou flou avec la décision...) en fournissant une aide méthodologique à la conduite de ce type de processus en vue de la construction d'accords de régulation opératoires.

\section{RÉFÉRENCES}

ALLAIN Sophie (2004), La négociation comme concept analytique central d'une théorie de la régulation sociale, Négociations, 2004/2, p.23-40.

ALLAIN Sophie (2005a), "La médiation environnementale comme système de régulation politique. Application au gouvernement de l'eau ", in Faget J. (dir.), Médiation et action publique. La dynamique du fluide, Presses Universitaires de Bordeaux, Bordeaux, p. 135-150

ALLAIN Sophie (2005b), « Décider de l'opportunité d'un barrage-réservoir ou construire une négociation territoriale explicite de la régulation politique du domaine de l'eau ? ", in Allain Sophie (coord.), Grujard J., Raulet-Croset N., Décisions et délibérations dans les projets de barrage-réservoir vis-à-vis de la régulation politique du domaine de l'eau, Rapport final pour le programme "Concertation, Décision, Environnement » du Ministère de l'Environnement.

ALLAIN Sophie (2007), « La conduite d'un débat public sur un projet d'infrastructure : une activité de médiation spécifique. Réflexions à partir du débat public « Francilienne ", in Revel et alii, opus cité, p. 112-122.

ALLAIN Sophie (2008a), Négocier le bien commun territorial. Esquisse d'une sociologie de médiation, Mémoire d'Habilitation à Diriger les Recherches en Sociologie de I'Institut d'Etudes Politiques de Paris, Paris.

AlLAIN Sophie (2008b), « Le débat public : outil pour une négociation territoriale ? ", in Blanc N., Bonin S. (Eds.), Grands barrages et habitants. Les risques sociaux du développement, Paris, Editions QUAE, coll. Natures sociales, Paris, p. 225-242. 
BLATRIX Cécile (2007), « Genèse et consolidation d'une institution: le débat public en France ", in Revel et alii, opus cité, p. 43-56.

COMmAILlE Jacques et Bruno JOBERT (Ed.), 1998, Les métamorphoses de la régulation politique, Paris, L.G.D.J., Maison des Sciences de l'Homme.

DEWEY John (2003a), CEuvres philosophiques. I - Reconstruction en philosophie. Université de Pau, Farrago, Ed. Léo Scherrer.

DEWEY John (2003b), CEuvres philosophiques. II - Le public et ses problèmes. Université de Pau, Farrago, Ed. Léo Scherrer.

DuPONT Christophe (1994), La négociation. Conduite, théorie, applications, Paris, Dalloz, $4^{\text {è }}$ éd.

ELSTER Jon (Ed.) (1998), Deliberative Democracy, Cambridge, Cambridge University Press.

FriedBerg Erhard. (1993), Le Pouvoir et la Règle : Dynamiques de l'action organisée, Paris, Seuil.

HABERMAS Jürgen (1987), Théorie de l'agir communicationnel, Tome I, Paris, Fayard. HABERMAS Jürgen (1997), Droit et démocratie. Entre faits et normes, Paris, Gallimard.

LAVELLE Sylvain (2007), « La politique de la discussion. La dynamique du débat entre conversation et négociation ", in Revel et alii, opus cité, p. 353-366.

LECUYER Betrand-Pierre (1986), "Régulation sociale, contrôle social et "social control" », Revue Française de Sociologie, 1, vol. VIII, janv.-mars 1986.

LIPPMANN Walter (1993), The Phantom Public, Transaction Publishers, US.

MAGNETTE Paul (2004), «La Convention européenne : argumenter et négocier dans une assemblée constituante multinationale », Revue Française de Science Politique, vol. $54, \mathrm{n}^{\circ} 1$.

MANIN Bernard (entretien avec) (2002), "L'idée de démocratie délibérative dans la science politique contemporaine », in Blondiaux L., Sintomer Y. (coord.), Démocratie et délibération, Politix, Vol. 15, n57, p. 37-55.

MILLET Gibert (1981), La Stratégie du Verbe, Paris, Dunod.

Pruitt Dean et Peter Carnevale (1993), Negotiation in social conflict, Buckingham (U.K), Open University Press.

REMER Gary (2000), « Two Models of Deliberation : Oratory and Conversation in Ratifying the Constitution », The Journal of Political Philosophy, vol. 8, ํ1, p. 68-90.

Revel Martine, Blatrix Cécile, Blondiaux Loïc, Fourniau Jean-Michel, Herard-DuBREUIL Bertrand et Rémi LEFEBVRE (2007), Le débat public : une expérience française de démocratie participative, Paris, La Découverte.

SCHELLING Thomas (1986), Stratégie du conflit, Paris, PUF.

SIMARD Louis (2006), "L'apprentissage comme ressource dans la conduite des projets : quels effets sur le débat public ? », in Simard L., Lepage L., Fourniau J.M., Gariépy M. et Gauthier M., Le débat public en apprentissage, Paris, L'Harmat$\tan$, p.119-131.

URFALINO Philippe (2005), «La délibération n'est pas une conversation », Négociations, $\mathrm{n}^{\circ} 2005 / 2$, p. 99-114.

WALton Richard et Robert MCKERsie (1965), A Behavioral Theory of Labor Negotiations, New York, ILR Press, Ithaca.

ZARTMAN William (Ed.) (1994), International Multilateral Negotiation, San Francisco (CA), the Jossey-Bass Inc. 